\title{
Adverse Pregnancy Outcomes of Patients with History of First-Trimester Recurrent Spontaneous Abortion
}

\author{
Jing Yang, ${ }^{1}$ Yan Wang, ${ }^{1}$ Xiao-ye Wang, ${ }^{1}$ Yan-yu Zhao, ${ }^{1}$ Jing Wang, ${ }^{2}$ and Yang-yu Zhao ${ }^{1}$ \\ ${ }^{1}$ Obstetrics \& Gynecology Department, Peking University Third Hospital, Beijing 100191, China \\ ${ }^{2}$ Obstetrics \& Gynecology Department, Peking University International Hospital, Beijing 102206, China \\ Correspondence should be addressed to Yang-yu Zhao; zhaoyangyu001@163.com
}

Received 17 April 2017; Revised 15 June 2017; Accepted 18 June 2017; Published 17 July 2017

Academic Editor: Marco Scioscia

Copyright (c) 2017 Jing Yang et al. This is an open access article distributed under the Creative Commons Attribution License, which permits unrestricted use, distribution, and reproduction in any medium, provided the original work is properly cited.

\begin{abstract}
Although a history of first-trimester recurrent spontaneous abortion (FRSA) is regarded as a risk factor in antenatal care, the characteristic of subsequent pregnancy outcome is not clearly elucidated. Here, a retrospective analysis was performed on the clinical data of 492 singleton pregnant women. 164 of them with the history of FRSA were enrolled in study group, compared to 328 deliveries without the history of FRSA. For maternal outcomes, patients in the study group delivered earlier with mean gestational age and the incidences of cesarean section and postpartum hemorrhage were higher compared to the control group. For placental outcomes, the incidence of placenta-mediated pregnancy complications (PMPC) in the study group increased in terms of late-onset preeclampsia, oligohydramnios, early-onset fetal growth restriction, and second-trimester abortion. Patients in the study group were more likely to suffer from placenta accreta, placenta increta, and placenta percreta. For perinatal outcomes, the proportion of birth defects of newborns in the study group was greater. At last, logistic regression analyses showed that the history of FRSA was an independent risk factor for cesarean section and pregnancy complications. In conclusion, women with the history of FRSA are often exposed to an elevated incidence of maternal-placental-perinatal adverse pregnancy outcomes.
\end{abstract}

\section{Introduction}

Recurrent spontaneous abortion (RSA) refers to the consecutive occurrence of fetal loss (body weight $<1000 \mathrm{~g}$ ) happening more than 2 times before 28 gestational weeks with the same sex partner [1]. The incidence ranges from $2 \%$ to $4 \%$ [2], of which nearly $80 \%$ appear in the first trimester. First-trimester RSA (FRSA) occurs within 12 weeks of pregnancy and its pathogenesis is more complicated than second-trimester RSA. No etiologic factor is identified in approximately $50 \%$ of FRSA cases. Previous studies have revealed that women with a history of RSA are exposed to higher rates of adverse maternal and fetal outcomes in their subsequent pregnancies [3-5], but few studies have focused on FRSA. Due to high incidence and complex etiology of FRSA, it is of great importance to investigate the subsequent pregnancy outcomes in order to ensure an effective perinatal care. In this study, we analyzed the associations between maternal-placentalperinatal pregnancy outcomes and history of FRSA. No intervention was administered during their pregnancies. The aim was to provide better therapies for future perinatal care.

\section{Materials and Methods}

2.1. General Information. A retrospective population-based analysis of all singleton pregnant women who gave birth at Peking University Third Hospital between January 2010 and September 2013 was performed. Total delivery number was 12811 during this period. All clinical records/information were anonymized and deidentified prior to analysis. Comparisons were made between women who have and have not undergone unexplained FRSA in order to estimate the relationship between FRSA and adverse pregnancy outcomes.

2.2. Inclusion Criteria. All patients in the study group met the following criteria: patients who had $\geq 2$ abortions during early pregnancy. Furthermore, chromosome karyotypes of the couples were normal, and patients had no abnormal 
reproductive tract anatomy. Thyroid, endocrine functions and the insulin level were normal. In addition, TORCH IgM was negative, antiphospholipid and antinuclear antibodies were negative, and the coagulation function was normal. Furthermore, cervical secretion was negative. The couples were also found to be negative for hepatitis $B$, hepatitis C, syphilis, AIDS, or other infectious diseases. In addition, blocking antibodies was not detected among patients with normal results in the above examinations. A total of 164 cases were included. From 12647 cases without the history of FRSA and delivering in the same period, 328 patients were randomly selected at $1: 2$ ratio as the control group.

2.3. Exclusion Criteria. Exclusion criteria of all subjects included medical complications such as chronic hypertension, chronic kidney diseases, autoimmune diseases, and severe late-term pregnancy complications transferred from other hospitals.

2.4. Data Collection. Data were collected from the computerized perinatal database of the hospital, which comprised obstetric and perinatal information recorded directly after delivery by three obstetricians. Xiao-ye Wang and Yan-yu Zhao examined the information routinely before entering it into the database. Coding was done after assessing the medical prenatal care records as well as the routine hospital documents. These procedures assured the completeness and accuracy of the database. The following clinical characteristics were evaluated: gravidity, spontaneous abortion times, proportion of primiparous women, body mass index (BMI), gestational age at delivery, birth weight, the use of assisted reproductive therapy, and times of other adverse pregnancy histories. The following maternal complications were examined: the cesarean section and postpartum hemorrhage. Placental-derived disease including PMPC and placental diseases were assessed. PMPC refers to a group of maternal complications caused by insufficient placental implantation and placental ischemia, including preeclampsia, fetal growth restriction, placental abruption, spontaneous premature birth, and intrauterine fetal death [6-8]. Preeclampsia (PE) was subdivided into early-onset and lateonset $\mathrm{PE}$ according to the incidence of gestational age. 32 weeks was identified as the diagnostic cutoff between earlyonset FGR and late-onset FGR $[9,10]$. No intrauterine fetal death occurred in both groups. Preterm birth was defined as the birth of a live baby at $<37$ completed weeks of gestation. Very preterm birth was defined as the birth of a live baby at $<32$ weeks of gestation. Oligohydramnios caused by fetal malformations and maternal premature rupture of membranes was excluded. Placental-derived disease refers to placenta previa, placenta accreta, placenta increta, and placenta percreta. The following perinatal outcomes were assessed: small for gestational age (SGA), perinatal death, severe asphyxia neonatorum, and birth defects of newborns.

2.5. Statistical Analysis. Data were analyzed using Statistical Package for Social Sciences (SPSS version 19.0). Continuous data of normal distribution was presented as mean and standard deviation (mean $\pm \mathrm{SD}$ ). Independent sample $t$-test
TABLE 1: Comparison of clinical characteristics between the two groups.

\begin{tabular}{lccc}
\hline Characteristics & $\begin{array}{c}\text { Study group } \\
(N=164)\end{array}$ & $\begin{array}{c}\text { Control } \\
\text { group } \\
(N=328)\end{array}$ & $P$ value \\
\hline Maternal age (years) & $33.97 \pm 4.25$ & $30.48 \pm 3.65$ & $<0.001$ \\
Gravidity & $3.9 \pm 1.1$ & $2.2 \pm 1.0$ & $<0.001$ \\
Gestational age (w) & $35.7 \pm 5.2$ & $38.2 \pm 2.1$ & $<0.05$ \\
$\begin{array}{l}\text { Birth weight (g) } \\
\text { Body mass index }\end{array}$ & $2676 \pm 1014$ & $3245 \pm 70$ & $<0.001$ \\
$\begin{array}{l}\text { Proportion of } \\
\text { primiparous women }\end{array}$ & $145(88.4 \%)$ & $283(86.2 \%)$ & $>0.05$ \\
$\begin{array}{l}\text { Received ART } \\
\text { Other adverse pregnancy }\end{array}$ & $17(10.3 \%)$ & $26(7.9 \%)$ & $>0.05$ \\
histories & $11(6.7 \%)$ & $19(5.8 \%)$ & $>0.05$ \\
\hline
\end{tabular}

was used for comparison between the two groups. Chi-square test was performed to ascertain differences in qualitative variables. A multivariate logistic regression analysis was carried out in a backward elimination fashion to analyze the relationship between FRSA and adverse outcomes. $P<0.05$ was considered to be statistically significant.

\section{Results}

3.1. Baseline Characteristics of the Study Population. The study population included a total of 12811 singleton patients who delivered at Peking University Third Hospital during nearly 3-year study period, of which 164 (1.28\%) met the inclusion criteria. A total of 164 included cases remained for analysis. Gravidity and maternal age were significantly higher in the study group, while the gestational weeks at delivery and birth weight of newborns were less (Table 1).

3.2. Maternal Pregnancy Outcomes. Maternal pregnancy outcomes are presented in Table 2. There were significantly higher rates of cesarean section and postpartum hemorrhage.

3.3. Placenta Diseases. Placenta diseases are summarized in Table 3. PMPC such as the late-onset PE, oligohydramnios, early-onset FGR, and second-trimester abortion were significantly higher in the study group. There was no significant difference between the two groups in the incidence of earlyonset PE, late-onset FGR, placental abruption, and early and late preterm delivery. The incidences of placenta diseases such as placenta accreta, placenta increta, and placenta percreta were significantly higher in the study group $(P<0.05)$; the difference of placenta previa rate between the two groups was of no significance. Considering that maternal age could cause the different incidence of late-onset PE, we then performed a multivariate logistic regression analysis in adjusted model and found that the incidence of late-onset PE was also significantly higher in the study group (Table 4).

3.4. Adverse Fetal and Neonatal Outcomes. Adverse perinatal outcomes are presented in Table 5. The proportion of birth defects of newborns in the study group was significantly 
TABLE 2: Comparison of maternal pregnancy outcomes between the two groups.

\begin{tabular}{|c|c|c|c|c|c|}
\hline Characteristics & $\begin{array}{c}\text { Study group } \\
N(\%)\end{array}$ & $\begin{array}{c}\text { Control group } \\
N(\%)\end{array}$ & OR & $95 \%$ CI & $P$ value \\
\hline Cesarean section & $105(64.02)$ & $152(46.34)$ & 2.06 & $1.40-3.03$ & $<0.001$ \\
\hline Postpartum hemorrhage & $17(10.37)$ & $18(5.49)$ & 1.99 & $0.10-3.98$ & $<0.05$ \\
\hline
\end{tabular}

TABLE 3: Comparison of placenta diseases between the two groups.

\begin{tabular}{|c|c|c|c|c|c|}
\hline Characteristics & $\begin{array}{c}\text { Study group } \\
N(\%)\end{array}$ & $\begin{array}{c}\text { Control group } \\
N(\%)\end{array}$ & OR & $95 \% \mathrm{CI}$ & $P$ value \\
\hline \multicolumn{6}{|c|}{ Placenta-derived maternal complications } \\
\hline Early-onset PE & $7(4.27)$ & $6(1.83)$ & 2.39 & $0.791-7.24$ & 0.196 \\
\hline Late-onset PE & $17(10.37)$ & $9(2.74)$ & 4.1 & $1.79-9.41$ & 0.002 \\
\hline Oligohydramnios & $17(10.37)$ & $7(2.13)$ & 5.3 & $2.15-13.06$ & $<0.001$ \\
\hline Early-onset FGR & $8(4.88)$ & $2(0.61)$ & 8.36 & $1.75-39.83$ & 0.005 \\
\hline Late-onset FGR & $3(1.83)$ & $5(1.52)$ & 1.2 & $0.28-5.10$ & 1 \\
\hline Placental abruption & $3(1.83)$ & $1(0.30)$ & 6.09 & $0.639-59.04$ & 0.241 \\
\hline Late abortion & $16(9.76)$ & $3(0.91)$ & 11.71 & $3.36-40.81$ & $<0.001$ \\
\hline Preterm birth & $8(4.88)$ & $8(2.44)$ & 2.051 & $0.76-5.57$ & 0.15 \\
\hline Very preterm birth & $14(8.54)$ & $23(7.01)$ & 1.24 & $0.62-2.47$ & 0.546 \\
\hline \multicolumn{6}{|l|}{ Placenta diseases } \\
\hline Placenta previa & $21(12.80)$ & $30(9.15)$ & 1.46 & $0.81-2.64$ & 0.209 \\
\hline Placenta accreta & $22(13.41)$ & $9(2.74)$ & 5.49 & $2.47-12.23$ & $<0.001$ \\
\hline $\begin{array}{l}\text { Placenta increta and } \\
\text { placenta percreta }\end{array}$ & $7(4.27)$ & $2(0.61)$ & 7.268 & $1.49-35.39$ & 0.012 \\
\hline
\end{tabular}

TABLE 4: Comparison of late-onset PE between the two groups.

\begin{tabular}{|c|c|c|c|c|c|c|}
\hline \multirow{3}{*}{ Variable } & \multicolumn{6}{|c|}{ Late-onset PE } \\
\hline & & Unadjusted & & & Adjusted & \\
\hline & OR & $95 \% \mathrm{CI}$ & $P$ value & OR & $95 \% \mathrm{CI}$ & $P$ value \\
\hline Maternal age & 4.1 & $1.79-9.41$ & 0.002 & 3.2 & $1.34-7.63$ & 0.009 \\
\hline
\end{tabular}

TABLE 5: Comparison of adverse fetal and neonatal outcomes between the two groups.

\begin{tabular}{lcccc}
\hline Characteristics & $\begin{array}{c}\text { Study group } \\
N(\%)\end{array}$ & $\begin{array}{c}\text { Control group } \\
N(\%)\end{array}$ & OR & 95\% CI \\
\hline Small for gestational age & $7(4.27)$ & $5(1.52)$ & 2.88 & $0.90-9.22$ \\
Perinatal death & $3(1.83)$ & $2(0.61)$ & 3.04 & 0.121 \\
Neonatal severe asphyxia & $1(0.61)$ & $1(0.30)$ & 2.01 & 0.427 \\
Birth defects of newborns & $12(7.32)$ & $3(0.91)$ & 8.55 & $0.13-32.28$ \\
\hline
\end{tabular}

higher as compared to the control group. There was no significant difference in the incidence of small for gestational age, perinatal death, and neonatal severe asphyxia between two the groups.

3.5. Adverse Pregnancy Outcomes Related to Recurrent Spontaneous Abortions. Using a multivariate regression analysis, with the following conditions as the outcome variable, respectively, controlling for corresponding confounding variables, FRSA was found as an independent risk factor for the following adverse pregnancy outcomes: cesarean section $(\mathrm{OR}=1.7$;
95\% CI: 1.06-2.73; $P<0.05)$ and placenta diseases including $\mathrm{PE}(\mathrm{OR}=3.69 ; 95 \% \mathrm{CI}: 1.87-7.30 ; P<0.001)$, oligohydramnios (OR $=4.62$; 95\% CI: $1.84-11.64 ; P=0.001)$, and second-trimester abortion $(\mathrm{OR}=11.71 ; 95 \% \mathrm{CI}$ : 3.36-40.81; $P<0.001$ ) (Table 6).

\section{Discussion}

Most experts believe that women with a history of two or three consecutive spontaneous abortions have similar abortion risks in their subsequent pregnancies. Patients who 
TABLE 6: Adverse pregnancy outcomes related to recurrent spontaneous abortions.

\begin{tabular}{|c|c|c|c|c|c|c|}
\hline Adverse outcomes & $\begin{array}{c}\text { Regression coefficient } \\
\beta \\
\end{array}$ & Standard error & $\begin{array}{c}\text { Wald } \\
\chi^{2}\end{array}$ & $P$ & OR & $95 \%$ CI \\
\hline $\mathrm{PE}$ & 1.306 & 0.348 & 14.102 & 0 & 3.69 & $1.87-7.30$ \\
\hline Oligohydramnios & 1.531 & 0.471 & 10.565 & 0.001 & 4.62 & $1.84-11.64$ \\
\hline Late abortion & 2.461 & 0.637 & 14.925 & 0 & 11.71 & $3.36-40.81$ \\
\hline Cesarean section & 0.531 & 0.242 & 4.825 & 0.028 & 1.7 & $1.06-2.73$ \\
\hline
\end{tabular}

experienced more than 2 incidents of miscarriage are often exposed to elevated incidences of placental dysfunction disorders and cesarean section $[3,11]$. Such patients should be considered as high risk obstetric population. Their characteristics of pregnancy complications and the preventive measures should be investigated due to the necessity and critical clinical value. However, limitation of current studies cannot exclude confounding effects of other pathologies when examining pregnancy outcomes considering complex causes of recurrent miscarriage. The unexplained miscarriage group is the best group minimizing other confounding factors and therefore it is the most appropriate group [5]. In this study, only patients who experienced two or more unexplained spontaneous abortions were enrolled; they also received no special perinatal interventions during obstetric examinations, which can minimize the impact of different abortion causes and perinatal treatments. The characteristics of adverse pregnancy outcomes were studied from a placentalperinatal-maternal perspective. We found that placentalderived pregnancy-related complications and cesarean section rate were significantly correlated with FRSA history. Specific perinatal care and corresponding preventions of placenta diseases can be implemented to reduce the incidence of adverse pregnancy outcomes.

4.1. Placenta-Derived Diseases. In previous studies, RSA has been reported to have a similar pathogenesis to that of placental dysfunction complications, such as preeclampsia, intrauterine fetal death, oligohydramnios, small for gestational age children, placental abruption, and spontaneous preterm birth. It also correlates with placental ischemia caused by placenta implantation insufficiency during the first trimester [12-14], immune imbalance of Thl/Th2 and Thl7/Treg on maternal-fetal interface, insufficient invasion of extravillous trophoblastic cells, and uterine spiral artery dysfunction. Although the late-term clinical manifestations differ, these types of diseases may have a common foundation [15]. Some scholars have named them as placental ischemic diseases [16]. Pregnancy outcome varies according to the extent of spiral artery remodeling. Partial remodeling disorder is prone to be complicated by preterm birth and FGR without pregnancy-induced hypertension; complete remodeling disorder is often accompanied with PE [17]. Placenta dysfunctions in different pregnancy stages also indicate different outcomes, such as infertility, spontaneous abortion, RSA, late abortion, spontaneous preterm birth (no rupture of membranes and premature rupture of membranes), PE, and FGR [18]. Gunnarsdottir et al. [11] have confirmed that patients with a history of more than two abortions were exposed to an increasing risk of placenta-derived pregnancyrelated complications, which is consistent with our study results. In terms of FGR, the pathogenesis between earlyonset and late-onset FGR differs. The early-onset FGR is caused by reduced density of trophoblastic vessels and impaired invasion ability in the second trimester [19], which is similar to that of FRSA. In this study, patients with FGR were subgrouped by their gestational weeks at the first diagnosis. We discovered that patients who suffered from FRSA had a significantly higher risk of early-onset FGR. Previous studies have shown that patients with early-onset PE may have early placental implantation defects and spiral artery remodeling disorders, further leading to placental ischemia. Late-onset PE is often related to maternal basic conditions, such as cardiovascular diseases and metabolic diseases [20]. Therefore, early-onset $\mathrm{PE}$ and recurrent miscarriage are more closely related. Although the incidences of early-onset and late-onset $\mathrm{PE}$ in study group were both higher than those in the control group, the difference in early-onset PE was not statistically significant. In view of the low incidence of PE and the limited sample size, further enrollment of PE patients is needed to identify the relationship between FRSA and earlyonset PE.

History of multiple abortions and abortion curettages are confirmed to be high risk factors of placenta previa and placenta accreta [21]. But the relationship between gestational hypertension and placenta previa remains controversial. A meta-analysis by Ying et al. [22] revealed that patients with gestational hypertension have a reduced risk of placenta previa. In this study, no significant difference of placenta previa occurrence was observed between the two groups. Maybe increased incidence of PE provides some protective factors for placenta previa. Usta et al. [23] suggest that a history of multiple pregnancy will affect the incidence of placenta accreta. In this study, the incidences of placental accreta and placental increta/percreta were higher in the study group than in the control group, which is consistent with those previous studies.

4.2. Maternal Pregnancy Outcomes. This study shows that RSA is an independent risk factor for cesarean section, which is consistent with previous studies [3]. In our study group, the age differences and history of abortion lead to no-indication cesarean sections. Placenta previa, placenta implantation/penetration, and FGR raised the incidence of cesarean sections with absolute indications. Postpartum hemorrhage rate was significantly higher in the study group than in the control group. Previous studies have shown that $\mathrm{PE}$ is an independent risk factor for uterine contraction 
fatigue and postpartum hemorrhage after vaginal delivery [24], and placental abnormalities (placenta previa, implantation, or early ablation) and hypertension are closely related to severe postpartum hemorrhage $[25,26]$.

4.3. Perinatal Outcomes. For FRSA, possibly due to the limitations of traditional cytogenetic techniques, no abnormal chromosomes in couples or previous fetus' chromosomal submicroscopic abnormalities were found. Increased incidence of villi genome microdeletions or microrepetition will lead to abortion or fetal chromosomal abnormalities in subsequent pregnancies [27]. Previous studies also revealed that subsequent pregnancies of FRSA patients were prone to fetal structural abnormalities $[28,29]$, especially for those with advanced maternal age and a history of more than three miscarriages. In this study, the proportion of neonatal birth defects in the study group increased accordingly.

Recent animal studies have shown that adverse factors in early and mid-term pregnancy affect pregnancy outcomes seriously, especially on the placenta and fetus. In lateterm pregnancy, maternal involvement is mainly affected by adverse factors [30]. It can be inferred that unexplained abortions and abortions of failed effective interventions might be affected prenatally and continued till mid-pregnancy. Placenta and fetus suffered the most, leading to placentalderived pregnancy-related complications, such as late-onset $\mathrm{PE}$, oligohydramnios, early-onset FGR, late abortion, placenta accreta, placenta increta, and placenta percreta. However, the numbers for the outcomes of early-onset FGR and placenta increta and placenta percreta are too small, and our further research will focus on those aspects.

The recent impact on fetuses is an increasing risk of perinatal death, FGR, small for gestational age fetus, and preterm birth [31, 32]. Current studies suggest that placenta dysfunctions have a profound impact on fetal long-term health, such as cardiovascular diseases, hypertension, obesity, nervous system dysfunctions, and psychological behavior abnormalities $[33,34]$. In this study, we mainly focused on the near-term influences on fetal health. The incidence of small for gestational age fetus, perinatal death, and neonatal severe asphyxia in the study group was higher than that in the control group, but the difference was not statistically significant. Small sample size, excellent capacity of prenatal care, and after-birth pediatric treatment for high risk pregnancies in our hospital may contribute to this situation. Enlarged sample size will be needed and long-term fetus health conditions should also be considered in future researches.

4.4. Innovations of This Study. To the best of our knowledge, it is the first study that subdivided PE and FGR patients to identify the correlations between FRSA and early-onset PE and FGR. In addition, the second-trimester abortion rate in patients who experienced FRSA is 18.059 times of that in those without previous FRSA. The current studies on maternal-fetal interface immunity revealed that both secondtrimester abortion and spontaneous preterm birth, similar to those of PE and FGR, suffered from abnormal immune cell function and cell number, leading to disorders of maternalfetal tolerance and uterine spiral artery reconstructions [26].
Recent reports have shown that about $50 \sim 65 \%$ of women with unexplained spontaneous abortion have at least one inherited or acquired prethrombotic state (PTS) [35]. In PTS, FVL mutation raises the risk of early miscarriages and early intrauterine fetal deaths [36]. Meta-analysis has shown that late spontaneous abortion is closely related to factors $\mathrm{V}$ and II (prothrombin) mutations and to the lack of protein $S$ induced congenital thrombosis [37]. Clinically, patients with RSA are not recommended for routine hereditary thrombosis test, in that mutations of factors V and II (prothrombin) in domestic Han population are rare. Therefore, tests of FVL, coagulation factor VIII, antithrombin, and protein C are only recommended for patients with a thrombosis family history and who suffered from unexplained FRSA. Late abortions should also be alerted.

4.5. Limitations of This Study. Sample selection bias in this retrospective study is inevitable. The diagnosis of unexplained abortion in the study group is exclusive. Due to the complexity of pathogenesis and the lack of specific clinical manifestations, FRSA diagnosis was mainly based on laboratory results. However, the test stability and standard are not unified among different laboratories, the exclusion of abortion causes was incomplete, and false positive diagnosis could not be avoided. Meanwhile, as our hospital is a university-affiliated high-class hospital and regional intense care referral center, patients in the control group could also be high risk pregnancies which to some extent weakened the difference between the two groups. In future study, with the standardization of RSA diagnosis and screening, a multicenter study should be performed for more objective and precise investigations on pregnancy outcomes.

For patients with a history of FRSA, this study shows an increased incidence of adverse maternal-placental-fetal outcomes for subsequent pregnancy, especially for placentaderived pregnancy-related complications. Late abortions, oligohydramnios, and $\mathrm{PE}$ are all independent risk factors for FRSA. For such pregnant women, perinatal care should focus on the monitoring of maternal-placental-fetal and neonatal conditions from prepregnancy to postpartum period. All of the therapies should be implemented both generally and individually. Specific steps are as follows: further exploration of abortion causes during prepregnancy or early pregnancy, especially for those with unexplained abortions; development of individualized prenatal care mode and monitoring treatment program in combination with maternal high risk factors and abortion check results; focusing on placentalfetal-maternal aspects as well as their interactions for perinatal care; individualized treatments for early intervention of related complications; dynamic monitoring measures and effective and safe treatments; effective prevention of adverse pregnancy outcomes during delivery; and postpartum monitoring and treatment and follow-up. All of the above can fundamentally reduce the incidence of mother and child complications and improve the adverse pregnancy outcomes.

\section{Conflicts of Interest}

The authors declare that they have no conflicts of interest. 


\section{Acknowledgments}

This work was supported by the National Key Research and Development Program of Reproductive Health \& Major Birth Defects Control and Prevention (Grant no. 2016YFC1000400).

\section{References}

[1] Practice Committee of American Society for Reproductive Medicine, "Definitions of infertility and recurrent pregnancy loss: a committee opinion," Fertility and Sterility, vol. 99, no. 1, article 63, 2013.

[2] J. L. Allison and D. J. Schust, "Recurrent first trimester pregnancy loss: Revised definitions and novel causes," Current Opinion in Endocrinology, Diabetes and Obesity, vol. 16, no. 6, pp. 446-450, 2009.

[3] E. Sheiner, A. Levy, M. Katz, and M. Mazor, "Pregnancy outcome following recurrent spontaneous abortions," European Journal of Obstetrics Gynecology and Reproductive Biology, vol. 118, no. 1, pp. 61-65, 2005.

[4] K. Field and D. J. Murphy, "Perinatal outcomes in a subsequent pregnancy among women who have experienced recurrent miscarriage: a retrospective cohort study," Human Reproduction, vol. 30, no. 5, pp. 1239-1245, 2015.

[5] M. Fawzy, S. Saravelos, T. C. Li, and M. Metwally, "Do women with recurrent miscarriage constitute a high-risk obstetric population?" Human Fertility, vol. 19, no. 1, pp. 9-15, 2016.

[6] R. Pijnenborg, L. Vercruysse, and I. Brosens, "Deep placentation," Best Practice \& Research: Clinical Obstetrics \& Gynaecology, vol. 25, no. 3, pp. 273-285, 2011.

[7] C. V. Ananth and A. M. Vintzileos, "Ischemic placental disease: epidemiology and risk factors," European Journal of Obstetrics Gynecology and Reproductive Biology, vol. 159, no. 1, pp. 77-82, 2011.

[8] R. W. Redline, "Placental pathology: a systematic approach with clinical correlations," Placenta, vol. 29, pp. S86-S91, 2008.

[9] S. Savchev, F. Figueras, M. Sanz-Cortes et al., "Evaluation of an optimal gestational age cut-off for the definition of early- and late-onset fetal growth restriction," Fetal Diagnosis and Therapy, vol. 36, no. 2, pp. 99-105, 2014.

[10] A. Nawathe and C. Lees, "Early onset fetal growth restriction," Best Practice \& Research Clinical Obstetrics \& Gynaecology, vol. 38, pp. 24-37, 2017.

[11] J. Gunnarsdottir, O. Stephansson, S. Cnattingius, H. Åkerud, and A.-K. Wikström, "Risk of placental dysfunction disorders after prior miscarriages: a population-based study," American Journal of Obstetrics and Gynecology, vol. 211, no. 1, pp. 34.e3134.e38, 2014.

[12] A. E. Whitten, R. Romero, S. J. Korzeniewski et al., "Evidence of an imbalance of angiogenic/antiangiogenic factors in massive perivillous fibrin deposition (maternal floor infarction): A placental lesion associated with recurrent miscarriage and fetal death," American Journal of Obstetrics and Gynecology, vol. 208, no. 4, pp. 310-el1, 2013.

[13] S. J. Benton, L. M. McCowan, A. E. P. Heazell et al., "Placental growth factor as a marker of fetal growth restriction caused by placental dysfunction," Placenta, vol. 42, pp. 1-8, 2016.

[14] S. Kwiatkowski, B. Dołęgowska, E. Kwiatkowska, R. Rzepka, A. Torbè, and M. Bednarek-Jędrzejek, "A common profile of disordered Angiogenic factor production and the exacerbation of inflammation in early preeclampsia, late preeclampsia, and intrauterine growth restriction," PLOS ONE, vol. 11, no. 10, Article ID e0165060, 2016.

[15] J. M. Roberts, "Pathophysiology of ischemic placental disease," Seminars in Perinatology, vol. 38, no. 3, pp. 139-145, 2014.

[16] C. V. Ananth, "Ischemic placental disease: a unifying concept for preeclampsia, intrauterine growth restriction, and placental abruption," Seminars in Perinatology, vol. 38, no. 3, pp. 131-132, 2014.

[17] I. Brosens, R. Pijnenborg, L. Vercruysse, and R. Romero, "The "great Obstetrical Syndromes" are associated with disorders of deep placentation," American Journal of Obstetrics \& Gynecology, vol. 204, no. 3, pp. 193-201, 2011.

[18] M. Hemberger, "Health during pregnancy and beyond: fetal trophoblast cells as chief co-ordinators of intrauterine growth and reproductive success," Annals of Medicine, vol. 44, no. 4, pp. 325-337, 2012.

[19] J. T. Henderson, E. P. Whitlock, E. O’Connor, C. A. Senger, J. H. Thompson, and M. G. Rowland, "Low-dose aspirin for prevention of morbidity and mortality from preeclampsia: a systematic evidence review for the U.S. Preventive Services Task Force," Annals of Internal Medicine, vol. 160, no. 10, pp. 695-703, 2014.

[20] E. A. Steegers, P. von Dadelszen, J. J. Duvekot, and R. Pijnenborg, "Pre-eclampsia," The Lancet, vol. 376, no. 9741, pp. 631644, 2010.

[21] T. Rosenberg, G. Pariente, R. Sergienko, A. Wiznitzer, and E. Sheiner, "Critical analysis of risk factors and outcome of placenta previa," Archives of Gynecology and Obstetrics, vol. 284, no. 1, pp. 47-51, 2011.

[22] H. Ying, Y. Lu, Y.-N. Dong, and D.-F. Wang, "Effect of placenta previa on preeclampsia," PLoS ONE, vol. 11, no. 1, Article ID e0146126, 2016.

[23] I. M. Usta, E. M. Hobeika, A. A. Abu Musa, G. E. Gabriel, and A. H. Nassar, "Placenta previa-accreta: risk factors and complications," The American Journal of Obstetrics and Gynecology, vol. 193, no. 3, pp. 1045-1049, 2005.

[24] L. A. Wetta, J. M. Szychowski, S. Seals, M. S. Mancuso, J. R. Biggio, and A. T. N. Tita, "Risk factors for uterine atony/postpartum hemorrhage requiring treatment after vaginal delivery," American Journal of Obstetrics and Gynecology, vol. 209, no. 1, pp. 51.e51-51.e56, 2013.

[25] M. S. Kramer, C. Berg, H. Abenhaim et al., "Incidence, risk factors, and temporal trends in severe postpartum hemorrhage," American Journal of Obstetrics and Gynecology, vol. 209, no. 5, pp. 449.e441-449.e447, 2013.

[26] A. Ekin, C. Gezer, U. Solmaz, C. E. Taner, A. Dogan, and M. Ozeren, "Predictors of severity in primary postpartum hemorrhage," Archives of Gynecology and Obstetrics, vol. 292, no. 6, pp. 1247-1254, 2015.

[27] B. Menten, K. Swerts, B. D. Chiaie et al., "Array comparative genomic hybridization and flow cytometry analysis of spontaneous abortions and mors in utero samples," BMC Medical Genetics, vol. 10, article no. 1471, p. 89, 2009.

[28] E. C. Larsen and O. B. Christiansen, "Recurrent miscarriage causes, disgnostics and treatment," Ugeskrift for Laeger, vol. 174, no. 41, pp. 2448-2452, 2012.

[29] R. B. Lathi, F. K. Gray Hazard, A. Heerema-Mckenney, J. Taylor, and J. T. Chueh, "First trimester miscarriage evaluation," Seminars in Reproductive Medicine, vol. 29, no. 6, pp. 463-469, 2011. 
[30] R.-Q. Ma, M.-N. Sun, and Z. Yang, "Effects of preeclampsia-like symptoms at early gestational stage on feto-placental outcomes in a mouse model," Chinese Medical Journal, vol. 123, no. 6, pp. 707-712, 2010.

[31] M. G. Walker, B. Fitzgerald, S. Keating, J. G. Ray, R. Windrim, and J. C. P. Kingdom, "Sex-specific basis of severe placental dysfunction leading to extreme preterm delivery," Placenta, vol. 33, no. 7, pp. 568-571, 2012.

[32] R. Romero, J. P. Kusanovic, T. Chaiworapongsa, and S. S. Hassan, "Placental bed disorders in preterm labor, preterm PROM, spontaneous abortion and abruptio placentae," Best Practice and Research: Clinical Obstetrics and Gynaecology, vol. 25, no. 3, pp. 313-327, 2011.

[33] R. M. Lewis, J. K. Cleal, and M. A. Hanson, "Review: placenta, evolution and lifelong health," Placenta, vol. 33, pp. S28-S32, 2012.

[34] J. N. Bulmer, G. J. Burton, S. Collins et al., "IFPA Meeting 2011 workshop report II: Angiogenic signaling and regulation of fetal endothelial function; Placental and fetal circulation and growth; Spiral artery remodeling," Placenta, vol. 33, pp. S9-S14, 2012.

[35] G. Mitić, A. Novakov Mikić, L. Povazan, A. Mitreski, V. Kopitović, and T. Vejnović, "Thromboprophylaxis implementation during pregnancy in women with recurrent foetal losses and thrombophilia," Medicinski Pregled, vol. 64, no. 9-10, pp. 471475, 2011.

[36] M. A. Rodger, M. T. Betancourt, P. Clark et al., "The association of factor $\mathrm{V}$ leiden and prothrombin gene mutation and placenta-mediated pregnancy complications: a systematic review and meta-analysis of prospective cohort studies," PLoS Medicine, vol. 7, no. 6, Article ID e1000292, 2010.

[37] G. Kovalevsky, C. R. Gracia, J. A. Berlin, M. D. Sammel, and K. T. Barnhart, "Evaluation of the association between hereditary thrombophilias and recurrent pregnancy loss: a meta-analysis," Archives of Internal Medicine, vol. 164, no. 5, pp. 558-563, 2004. 


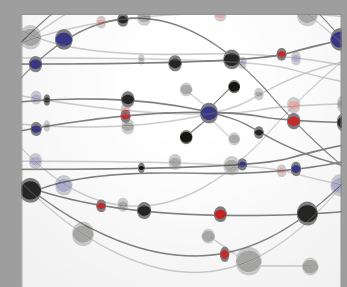

The Scientific World Journal
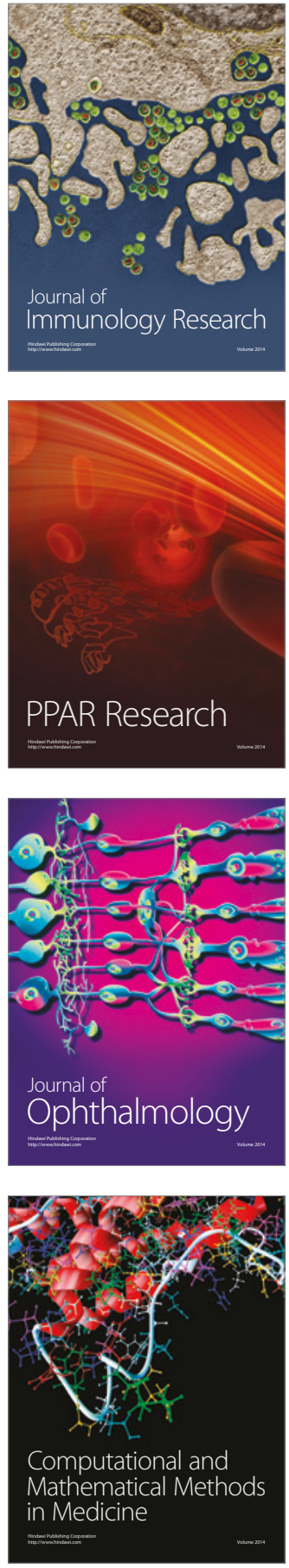

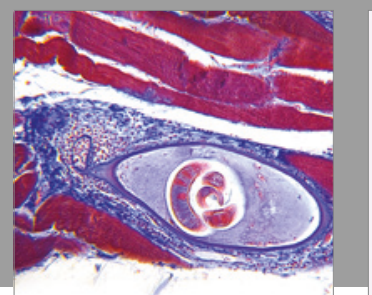

Gastroenterology Research and Practice
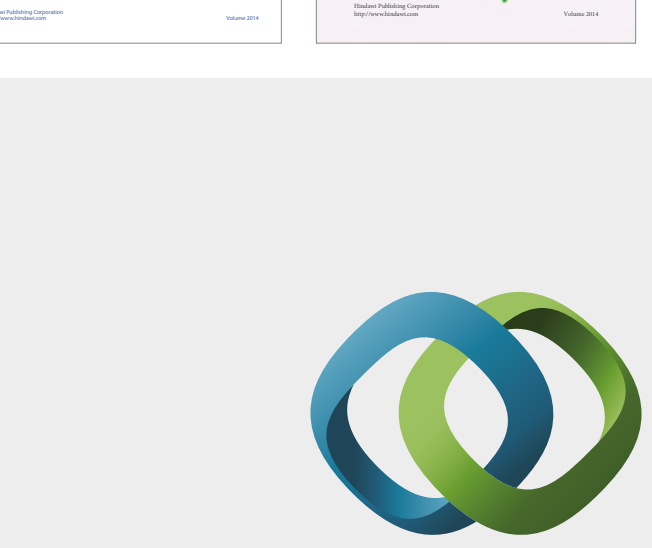

\section{Hindawi}

Submit your manuscripts at

https://www.hindawi.com
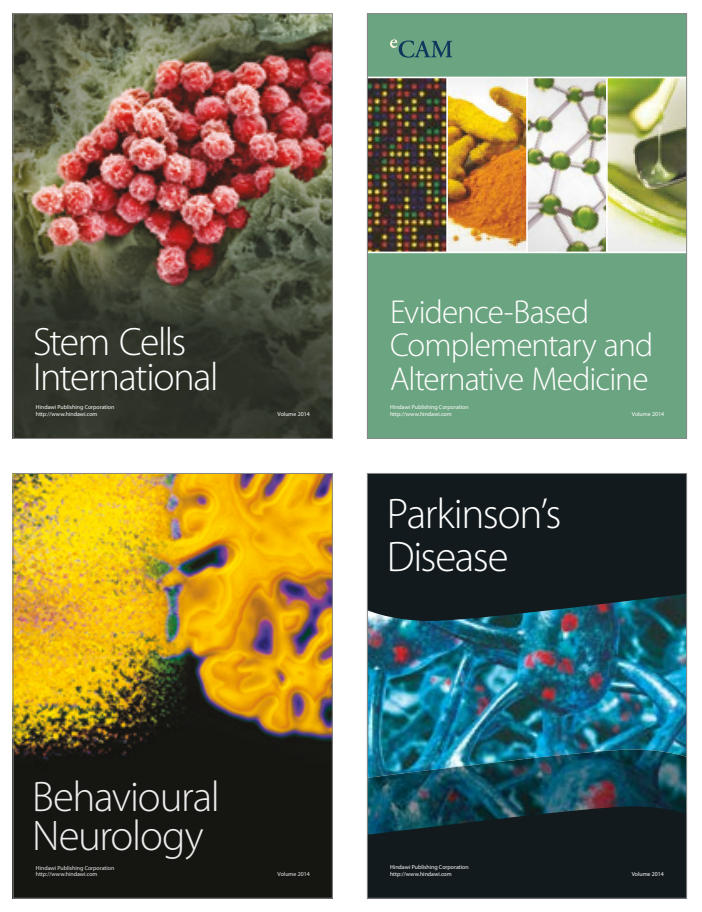
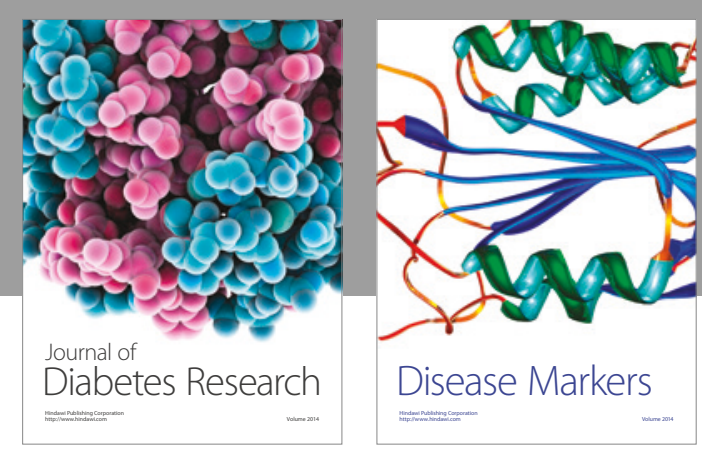

Disease Markers
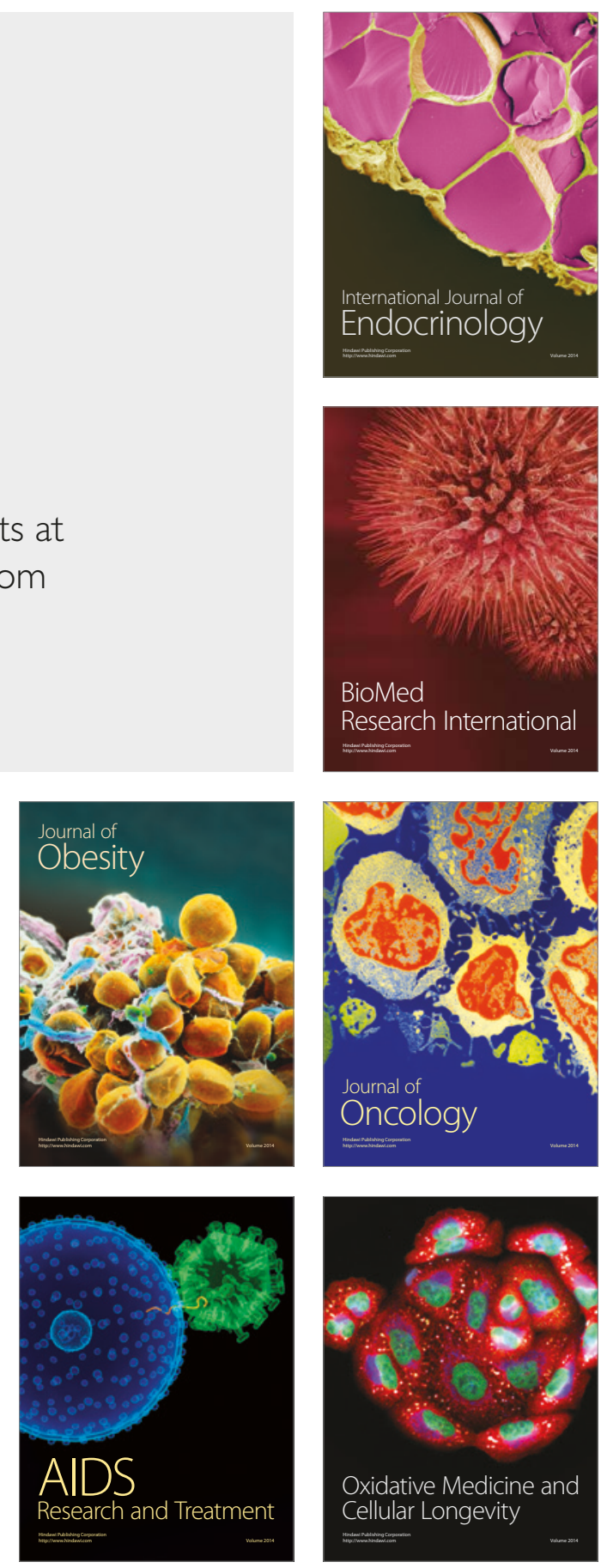